\title{
Selected patients can benefit more from the management of etoposide and platinum-based chemotherapy and thoracic irradiation-a retrospective analysis of 707 small cell lung cancer patients
}

\author{
Shoubo Cao ${ }^{1, *}$, Shi Jin ${ }^{1, *}$, Jing Shen ${ }^{1}$, Jingyan $\mathrm{Cao}^{1}$, Hua Zhang ${ }^{1}$, Qingwei Meng ${ }^{1}$, \\ Chunyan Wang ${ }^{1}$, Aiqi Zhang ${ }^{1}$, Pei Zhang ${ }^{1}$, Yan Yu ${ }^{1}$ \\ ${ }^{1}$ Department of Medical Oncology, Harbin Medical University Cancer Hospital, Harbin, China \\ * Shoubo Cao and Shi Jin contributed equally to this work and should be considered co-first authors \\ Correspondence to: Yan Yu, email: yuyan@ems.hrbmu.edu.cn \\ Keywords: small cell lung cancer, prognostic factors, subgroup analysis, inflammation, survival \\ Received: June 29, $2016 \quad$ Accepted: December 05, 2016 Published: December 31, 2016
}

\section{ABSTRACT}

The management of small cell lung cancer (SCLC) has reached a plateau. Etoposide and platinum-based chemotherapy plus thoracic irradiation remain the standard treatment strategy for SCLC. Our study aims to assess the potential prognostic factors of patients treated with etoposide and platinum-based chemotherapy and explore which group of patients can benefit more from standard treatment strategies. On univariate analysis, age $>65$ years, male patients, KPS (Karnofsky Performance Status) $\leq 80$ points, positive smoking history, anemia, lymphocyte counts $\leq 1.65 \times 10^{\circ} / \mathrm{L}$, neutrophil to lymphocyte ratio $(N L R)>3.18$, lymphocyte to monocyte ratio $(L M R) \leq 2.615$, lactate dehydrogenase $(L D H)>216.5 \mathrm{U} / \mathrm{L}$, alkaline phosphatase $(A L P)>119.5 \mathrm{U} / \mathrm{L}$, absence of surgery, absence of thoracic irradiation, chemotherapy cycles $<4$, metastatic sites $\geq 2$ and extensive disease were correlated with a poor prognosis. Gender, KPS, chemotherapy cycles, thoracic irradiation, metastatic sites, LDH and tumor stage held statistical significance on multivariate analysis $(p<0.05)$. High LDH was closely correlated with extensive disease, metastatic sites $\geq 2$, anemia, low LMR, high NLR and ALP levels. Subgroup analysis showed patients with male

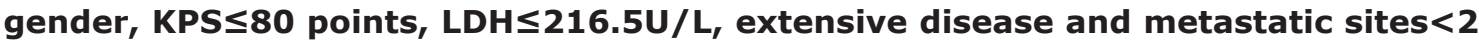
could benefit more from $\geq 4$ chemotherapy cycles. Patients with male gender, KPS $>80$ points, LDH $\leq 216.5 \mathrm{U} / \mathrm{L}$, limited disease and metastatic sites $<2$ could benefit more from thoracic irradiation ( $p<0.05$ on uni- and multivariate analysis). In conclusion, female patients, KPS $>80$ points, chemotherapy cycles $\geq 4$, thoracic irradiation, metastatic sites $<2$, LDH $\leq 216.5 \mathrm{U} / \mathrm{L}$ and limited disease were independent positive prognostic factors for SCLC patients treated with etoposide and platinum-based chemotherapy. Selected patients can benefit more from the management of $\geq 4$ cycles of chemotherapy and thoracic irradiation.

\section{INTRODUCTION}

SCLC is an aggressive disease with a high mortality and negative prognosis. Median survival is less than 1 year, with a 5-year survival of approximately $5 \%$ [1]. The management of SCLC has also reached a plateau for nearly 30 years. Etoposide and platinum-based chemotherapy plus thoracic irradiation remain the standard treatment strategy for SCLC [2]. However, treatment outcome is significantly different. Although patients received the same treatment strategies, many of them progressed quickly and even died soon. Systematic chemotherapy and thoracic radiotherapy have a limited role in these patients. On one hand, it leads to the increasing of economic burdens. On the other hand, it also adds some toxic reactions resulted from the treatment. The identification of patients who can 
benefit more from the treatment of chemotherapy and thoracic radiotherapy is of great value in clinics.

A number of retrospective studies have been performed to assess the prognostic roles of parameters in SCLC patients, but the patients enrolled are not treated with the same chemotherapy strategy and the results are not identical. Poor performance status, elevated serum LDH and extensive disease are usually associated with a short term survival in most studies [1, 3-5]. Alkaline phosphatase (ALP) [1, 5-8], age [9], gender [3, 8, 9], neutrophil [4, 9] and serum albumin $[4,6]$ were also found to be correlated with SCLC prognosis in some studies. The potential prognostic values of these parameters in SCLC patients treated with the same chemotherapy strategy remains unclear.

In clinics, we find the diagnosis of SCLC is usually accompanied by inflammation in most patients. Inflammation and the subsequent tumor development or tumor-elicited inflammation are critical steps for the development of cancers $[10,11]$. Inflammation is often regarded as a feature of body innate immunity [11]. All types of immune cells can exist in the core, invasive margin of tumors as well as the adjacent tertiary lymphoid structures, and they can directly or indirectly affect tumor development through the secretion of cytokines [1214]. Neutrophil to lymphocyte ratio (NLR), platelet to lymphocyte ratio (PLR) and lymphocyte to monocyte ratio (LMR), as key inflammatory markers, have been found to be associated with the prognosis of various malignancies including SCLC [15-21]. However, recent studies with small number of SCLC patients enrolled seldom assess the prognostic roles of some biochemical markers, lymphocyte, monocyte or LMR [16, 21]. And previous studies rarely involve the roles of lymphocyte, monocyte, NLR, PLR and LMR [1, 3-9]. We will take more serum biochemical markers into consideration to explore their prognostic values.

Our present study aims to assess the potential prognostic values of parameters in the presence of more serum biochemical markers in the whole group and variable subgroups of SCLC patients treated with the same chemotherapy strategy. But more importantly, we want to explore which group of patients can benefit more from the treatment of more cycles of etoposide and platinumbased chemotherapy and thoracic irradiation, and this is significant in clinics. It can help gain greater clinical benefits and survival time in the condition of smaller economic burdens and slighter toxic reactions.

\section{RESULTS}

\section{Patient characteristics}

This study included 707 patients (454 men, 253 women). The mean age was $56.24 \pm 10.15$ years (range $23-75)$. Smoking history was present in $62.5 \%(442 / 707)$ patients (Table 1). The most common metastatic sites at diagnosis were bone (58/707, 8.2\%), liver (47/707, 6.6\%), brain $(44 / 707,6.2 \%)$ and adrenal glands $(21 / 707,3.0 \%)$. The most common symptoms at presentation were cough (597/707, 84.4\%), shortness of breath $(244 / 707,34.5 \%)$ and chest pain $(243 / 707,34.4 \%)$. Of the 707 patients, $288(40.7 \%)$ patients suffered ED, 419 (59.3\%) patients suffered LD. All patients were treated with etoposide and platinum-based chemotherapy, and only 294 (41.6\%) patients received thoracic radiotherapy. The KPS was $\leq 80$ points in 164 cases $(23.2 \%)$ and $>80$ points in 543 cases $(76.8 \%)$. The median time from initial symptoms presented to definitive diagnosis was 1.0 months. The median survival time (MST) was 15.0 months (95\% CI, 13.552-16.448) for all cases and 145 patients were still alive at present (Figure 1). Of all 145 cases, 109 had LD and 36 had ED. The survival rates at 1-, 2- and 5-year were $59.0 \%, 34.1 \%$ and $20.8 \%$, respectively. The median survival time and 1-, 2-, 5-year survival rate for LD and ED were 21.0 months, $71.6 \%, 43.7 \%, 26.5 \%$ and 10.0 months, $40.6 \%, 20.1 \%, 12.5 \%$, respectively.

\section{The cutoff values for neutrophil, lymphocyte, monocyte, NLR, PLR, LMR, LDH and ALP}

The mean $( \pm \mathrm{SD})$ neutrophil, lymphocyte and monocyte counts, NLR, PLR, LMR, LDH and ALP were $4.55( \pm 2.01), 1.68( \pm 0.62), 0.49( \pm 0.23) \times 10^{9} / \mathrm{L}$, $3.16( \pm 2.44), 174.90( \pm 107.97), 5.72( \pm 15.43), 210.75$ $( \pm 136.20) \mathrm{U} / \mathrm{L}$ and $104.47( \pm 52.89) \mathrm{U} / \mathrm{L}$, respectively. The ROC analysis showed the optimal LDH cut-off was $216.5 \mathrm{U} / \mathrm{L}(p<0.001, \mathrm{AUC}, 0.611 ; 95 \% \mathrm{CI}, 0.569-0.652)$. The patients were then divided into high $(>216.5 \mathrm{U} / \mathrm{L})$ and low LDH ( $\leq 216.5 \mathrm{U} / \mathrm{L})$ groups. There were $192(27.2 \%)$ patients in the high group and $515(72.8 \%)$ in the low LDH group. The ALP of $119.5 \mathrm{U} / \mathrm{L}(p=0.231$, AUC, 0.526 ; $95 \%$ CI, $0.483-0.569)$, neutrophil of $4.95 \times 10^{9} / \mathrm{L}(p=0.577$, AUC, 0.512; 95\% CI, 0.469-0.555), lymphocyte of $1.65 \times 10^{9} / \mathrm{L}(p=0.009$, AUC, $0.557 ; 95 \%$ CI, 0.514-0.599), monocyte of $0.495 \times 10^{9} / \mathrm{L}(p=0.183$, AUC, $0.529 ; 95 \%$ CI, 0.486-0.571), NLR of $3.18(p=0.037$, AUC, $0.545 ; 95 \%$ CI, 0.503-0.588), PLR of $176.5(p=0.194$, AUC, 0.528; $95 \%$ CI, 0.486-0.571) and LMR of 2.615 ( $p=0.010$, AUC, 0.556 ; $95 \%$ CI, $0.513-0.598)$ were selected as the optimal cut-off values.

\section{Serum biochemical examinations}

Routine blood test and liver function evaluation were performed on each patient. The most common abnormalities were high levels of LMR (512/707,72.4\%), lymphocyte $(334 / 707,47.2 \%)$, monocyte $(321 / 707$, $45.4 \%)$, ALT $(268 / 707,37.9 \%)$, neutrophil (248/707, $35.1 \%)$, LDH $(192 / 707,27.2 \%)$, platelet (160/707, $22.6 \%)$, low albumin level $(105 / 707,14.9 \%)$ and anemia (64/707, 9.1\%). Carcinoembryonic antigen (CEA) and neuron specific enolase (NSE) were tested in 402 and 
Table 1: Clinical characteristics of patients with SCLC

\begin{tabular}{|c|c|c|}
\hline Features & $\mathbf{n}$ & $\%$ \\
\hline \multicolumn{3}{|l|}{ Age (years) } \\
\hline$\leq 65$ & 561 & 79.3 \\
\hline$>65$ & 146 & 20.7 \\
\hline \multicolumn{3}{|l|}{ Gender } \\
\hline Male & 454 & 64.2 \\
\hline Female & 253 & 35.8 \\
\hline \multicolumn{3}{|l|}{ Symptoms initially presented } \\
\hline Cough & 597 & 84.4 \\
\hline Shortness of breath & 244 & 34.5 \\
\hline Chest pain & 243 & 34.4 \\
\hline Hemoptysis or blood in phlegm & 233 & 33.0 \\
\hline Hoarseness & 30 & 4.2 \\
\hline \multicolumn{3}{|l|}{ KPS (Karnofsky Performance Status) } \\
\hline$>80$ point & 543 & 76.8 \\
\hline$\leq 80$ points & 164 & 23.2 \\
\hline \multicolumn{3}{|l|}{ Smoking history } \\
\hline Yes & 442 & 62.5 \\
\hline No & 265 & 37.5 \\
\hline \multicolumn{3}{|l|}{ Family history of cancer } \\
\hline Yes & 116 & 16.4 \\
\hline No & 591 & 83.6 \\
\hline \multicolumn{3}{|l|}{ Thoracic irradiation } \\
\hline Yes & 294 & 41.6 \\
\hline No & 413 & 58.4 \\
\hline \multicolumn{3}{|l|}{ Surgery } \\
\hline Yes & 25 & 3.5 \\
\hline No & 682 & 96.5 \\
\hline \multicolumn{3}{|l|}{ Chemotherapy cycles } \\
\hline$<4$ & 332 & 47.0 \\
\hline$\geq 4$ & 375 & 53.0 \\
\hline \multicolumn{3}{|l|}{ Metastatic sites } \\
\hline$<2$ & 642 & 90.8 \\
\hline$\geq 2$ & 65 & 9.2 \\
\hline \multicolumn{3}{|l|}{ Stage } \\
\hline LD & 419 & 59.3 \\
\hline ED & 288 & 40.7 \\
\hline
\end{tabular}


344 patients, and 29.1\% (117/402) and 86.9\% (299/344) patients showed an elevated level of CEA $(>5 \mathrm{ng} / \mathrm{ml})$ and NSE (>15.2 ng/ml), respectively.

\section{Univariate analysis}

Each parameter was assessed by univariate analysis. Significant prognostic factors were age $(p=0.025)$, gender $(p=0.034)$, KPS $(p<0.001)$, smoking history ( $p=0.009)$, anemia $(p=0.026)$, lymphocyte counts $(p=0.028)$, NLR $(p=0.002)$, LMR $(p=0.008)$, LDH $(p<0.001)$, ALP $(p=0.015)$, surgery $(p=0.011)$, thoracic irradiation $(p<0.001)$, chemotherapy cycles $(p<0.001)$, metastatic sites $(p<0.001)$ and tumor stage $(p<0.001)$. We also assessed the prognostic values of CEA $(n=402)$ and NSE ( $\mathrm{n}=344)$. High NSE level was a negative prognostic factor on univariate analysis ( $>15.2 \mathrm{vs} \leq 15.2 \mathrm{ng} / \mathrm{ml}, 14.0$ vs 24.0 months, $p=0.009$ ). No significant difference was found on variable CEA levels ( $>5 \mathrm{vs} \leq 5 \mathrm{ng} / \mathrm{ml}, 13.0$ vs 15.5 months, $p=0.219)$. These data showed the following factors were correlated with a poor prognosis: age $>65$ years, male patients, KPS $\leq 80$ points, positive smoking history, anemia (a hemoglobin value $<12.0 \mathrm{~g} / \mathrm{dl}$ in men and $11.0 \mathrm{~g} / \mathrm{dl}$ in women), lymphocyte counts $<1.65 \times 10^{9} / \mathrm{L}$, $\mathrm{NLR}>3.18, \mathrm{LMR} \leq 2.615, \mathrm{LDH}>216.5 \mathrm{U} / \mathrm{L}, \mathrm{ALP}>119.5$ $\mathrm{U} / \mathrm{L}, \mathrm{NSE}>15.2 \mathrm{ng} / \mathrm{ml}$, absence of surgery, absence of thoracic irradiation, chemotherapy cycles $<4$, metastatic sites $\geq 2$ and extensive disease (Table 2). The variables examined in the final multivariate analysis were age, gender, KPS, smoking history, anemia, lymphocyte counts, NLR, LMR, LDH, ALP, surgery, thoracic irradiation, chemotherapy cycles, metastatic sites and tumor stage.

\section{Multivariate analysis}

The multivariate analysis showed gender $(p=0.027)$, KPS $(p<0.001)$, LDH $(p=0.001)$, chemotherapy cycles $(p=0.005)$, thoracic irradiation $(p=0.013)$, metastatic sites $(p=0.015)$ and tumor stage $(p<0.001)$ were statistically significant (Table 3 ). The hazard ratios of death were observed for $\mathrm{LDH} \geq 216.5 \mathrm{U} / \mathrm{L}$ (1.415 fold increase compared to $\mathrm{LDH}<216.5 \mathrm{U} / \mathrm{L}), \mathrm{KPS} \leq 80$ points $(1.834$ fold increase compared to KPS $>80$ points), chemotherapy cycles $<4$ (1.276 fold increase compared to chemotherapy cycles $\geq 4)$, absence of thoracic irradiation ( 1.25 fold compared to the presence of thoracic irradiation), extensive disease (1.428 fold compared to limited disease). Female patients, KPS $>80$ points, $\mathrm{LDH} \leq 216.5 \mathrm{U} / \mathrm{L}$, chemotherapy cycles $\geq 4$, thoracic irradiation, metastatic sites $<2$ and limited disease were independent positive prognostic factors for long-term survival of SCLC patients treated with etoposide and platinum-based chemotherapy.

\section{The association between LDH and clinicopathological characteristics}

LDH was identified to be serum independent prognostic factor of the whole SCLC patients on uniand multivariate analysis. The optimal cutoff points to assess the difference of overall survival were $216.5 \mathrm{U} / \mathrm{L}$ for LDH. Using the optimal cutoff points, we divided all patients into two groups to evaluate the association of LDH levels and clinicopathological characteristics of SCLC patients (Table 4). We found that high levels of LDH was correlated with the presence of anemia (yes vs no, $39.1 \%$ vs $26.0 \%, p=0.025$ ) and high NLR (yes vs no,
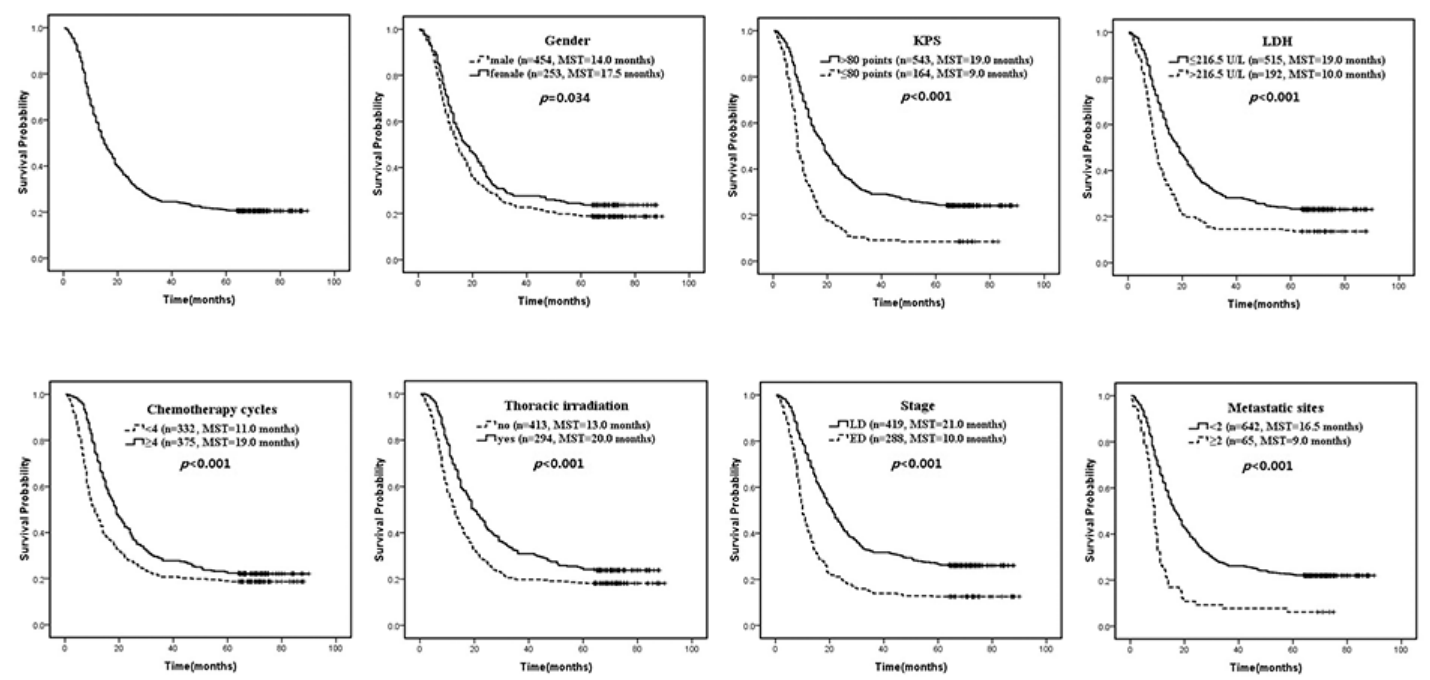

Figure 1: Overall survival and independent prognostic factors for 707 patients treated with etoposide and platinumbased chemotherapy. MST, median survival time. 
Table 2: Univariate analysis results of potential prognostic factors of SCLC patients treated with etoposide and platinum-based chemotherapy

\begin{tabular}{|c|c|c|c|c|c|}
\hline Variables & $\mathrm{O} / \mathrm{N}^{*}$ & $\%$ & median survival time (months) & $95 \%$ CI & p value \\
\hline Age (years) & & & & & 0.025 \\
\hline$\leq 65$ & $436 / 561$ & 77.7 & 16.0 & $14.129-17.871$ & \\
\hline$>65$ & $126 / 146$ & 86.3 & 15.0 & $12.787-17.213$ & \\
\hline Gender & & & & & 0.034 \\
\hline Male & $369 / 454$ & 81.3 & 14.0 & $12.394-15.606$ & \\
\hline Female & $193 / 253$ & 76.3 & 17.5 & $14.090-20.910$ & \\
\hline KPS & & & & & $<0.001$ \\
\hline$>80$ point & $412 / 543$ & 75.9 & 19.0 & $16.943-21.057$ & \\
\hline$\leq 80$ points & $150 / 164$ & 91.5 & 9.0 & $8.105-9.895$ & \\
\hline Smoking history & & & & & 0.009 \\
\hline Yes & $364 / 442$ & 82.4 & 14.0 & $12.640-15.360$ & \\
\hline No & $198 / 265$ & 74.7 & 18.5 & $15.367-21.633$ & \\
\hline $\begin{array}{l}\text { Family history of } \\
\text { cancer }\end{array}$ & & & & & 0.180 \\
\hline Yes & $98 / 116$ & 84.5 & 13.0 & $9.047-16.953$ & \\
\hline No & $464 / 591$ & 78.5 & 15.5 & 14.011-16.989 & \\
\hline Anemia & & & & & 0.026 \\
\hline Yes & $55 / 64$ & 85.9 & 11.5 & $8.070-14.930$ & \\
\hline No & $507 / 643$ & 78.8 & 16.0 & $14.469-17.531$ & \\
\hline $\mathrm{RBC}$ & & & & & 0.184 \\
\hline normal & $500 / 629$ & 79.5 & 16.0 & $14.485-17.515$ & \\
\hline Below normal & $62 / 78$ & 79.5 & 10.0 & $6.634-13.366$ & \\
\hline WBC $(\times 109 / \mathrm{L})$ & & & & & 0.135 \\
\hline$\leq 10$ & $522 / 652$ & 80.1 & 15.0 & $13.344-16.656$ & \\
\hline$>10$ & $40 / 55$ & 72.7 & 19.0 & $9.929-28.071$ & \\
\hline Platelet & & & & & 0.662 \\
\hline$\leq 300$ & $438 / 547$ & 80.1 & 15.0 & $13.472-16.528$ & \\
\hline$>300$ & $124 / 160$ & 77.5 & 14.5 & $11.143-17.857$ & \\
\hline Neutrophil $\left(\times 10^{9} / \mathrm{L}\right)$ & & & & & 0.518 \\
\hline$\leq 4.95$ & $364 / 495$ & 79.3 & 16.0 & $14.125-17.875$ & \\
\hline$>4.95$ & $198 / 248$ & 79.8 & 14.0 & $12.404-15.596$ & \\
\hline Lymphocyte $\left(\times 10^{9} / \mathrm{L}\right)$ & & & & & 0.028 \\
\hline$\leq 1.65$ & $303 / 373$ & 81.2 & 13.0 & $11.675-14.325$ & \\
\hline$>1.65$ & $259 / 334$ & 77.5 & 18.0 & $15.442-20.558$ & \\
\hline Monocyte $(\times 109 / \mathrm{L})$ & & & & & 0.117 \\
\hline$\leq 0.495$ & $303 / 386$ & 78.5 & 17.5 & $15.531-19.469$ & \\
\hline \multirow[t]{2}{*}{$>0.495$} & $259 / 321$ & 80.7 & 14.0 & $12.465-15.535$ & \\
\hline & & & & & (Continued) \\
\hline
\end{tabular}




\begin{tabular}{|c|c|c|c|c|c|}
\hline Variables & $\mathbf{O} / \mathbf{N}^{*}$ & $\%$ & median survival time (months) & $95 \% \mathrm{CI}$ & p value \\
\hline NLR & & & & & 0.002 \\
\hline$\leq 3.18$ & $357 / 462$ & 77.3 & 17.0 & 14.894-19.106 & \\
\hline$>3.18$ & $205 / 245$ & 83.7 & 13.0 & $11.014-14.986$ & \\
\hline PLR & & & & & 0.092 \\
\hline$\leq 176.5$ & $350 / 445$ & 78.7 & 17.0 & 15.211-18.789 & \\
\hline$>176.5$ & $212 / 262$ & 80.9 & 13.0 & $11.601-14.399$ & \\
\hline LMR & & & & & 0.008 \\
\hline$\leq 2.615$ & $161 / 195$ & 82.6 & 12.0 & $10.422-13.578$ & \\
\hline$>2.615$ & $401 / 512$ & 78.3 & 17.0 & $15.152-18.848$ & \\
\hline AST (U/L) & & & & & 0.194 \\
\hline$\leq 40$ & $495 / 623$ & 79.5 & 16.0 & $14.589-17.411$ & \\
\hline$>40$ & $67 / 84$ & 79.8 & 9.5 & $7.404-11.596$ & \\
\hline $\operatorname{ALT}(\mathrm{U} / \mathrm{L})$ & & & & & 0.574 \\
\hline$\leq 40$ & $351 / 439$ & 80.0 & 16.5 & $14.492-18.508$ & \\
\hline$>40$ & $211 / 268$ & 78.7 & 14.0 & $12.102-15.898$ & \\
\hline $\mathrm{LDH}(\mathrm{U} / \mathrm{L})$ & & & & & $<0.001$ \\
\hline$\leq 216.5$ & $396 / 515$ & 76.9 & 19.0 & $16.737-21.263$ & \\
\hline$>216.5$ & $166 / 192$ & 86.4 & 10.0 & $8.869-11.131$ & \\
\hline $\operatorname{ALP}(\mathrm{U} / \mathrm{L})$ & & & & & 0.015 \\
\hline$\leq 119.5$ & $431 / 547$ & 78.8 & 16.5 & $14.737-18.263$ & \\
\hline$>119.5$ & $131 / 160$ & 81.9 & 12.0 & $9.717-14.283$ & \\
\hline Serum albumin $(\mathrm{g} / \mathrm{L})$ & & & & & 0.378 \\
\hline$\leq 40$ & $85 / 105$ & 81.0 & 14.0 & $9.816-18.184$ & \\
\hline$>40$ & $477 / 602$ & 79.2 & 15.5 & $14.018-16.982$ & \\
\hline Globulin $(\mathrm{G}, \mathrm{g} / \mathrm{L})$ & & & & & 0.123 \\
\hline$\leq 35$ & $522 / 651$ & 80.2 & 15.0 & $13.626-16.374$ & \\
\hline$>35$ & $40 / 56$ & 71.4 & 20.0 & $12.055-27.945$ & \\
\hline Surgery & & & & & 0.011 \\
\hline Yes & $15 / 25$ & 60.0 & 31.0 & $0.000-69.353$ & \\
\hline No & $547 / 682$ & 80.2 & 15.0 & $13.707-16.293$ & \\
\hline Thoracic irradiation & & & & & $<0.001$ \\
\hline Yes & $224 / 294$ & 76.2 & 20.0 & $16.849-23.151$ & \\
\hline No & $338 / 413$ & 81.8 & 13.0 & $11.662-14.338$ & \\
\hline Chemotherapy cycles & & & & & $<0.001$ \\
\hline$<4$ & $270 / 332$ & 81.3 & 11.0 & $9.215-12.785$ & \\
\hline$\leq 4$ & $292 / 375$ & 77.9 & 19.0 & $16.919-21.081$ & \\
\hline
\end{tabular}

(Continued) 


\begin{tabular}{lccccc}
\hline Variables & $\mathbf{O} / \mathbf{N}^{*}$ & $\mathbf{\%}$ & median survival time (months) & $\mathbf{9 5 \%} \mathbf{C I}$ & $\mathbf{p}$ value \\
\hline $\begin{array}{l}\text { Metastatic sites } \\
<2\end{array}$ & $501 / 642$ & 78.0 & & & $<\mathbf{0 . 0 0 1}$ \\
$\leq 2$ & $61 / 65$ & 93.8 & 9.0 & $14.969-18.031$ & \\
Stage & & & & $8.022-9.978$ & \\
LD & $310 / 419$ & 74.0 & 21.0 & & $<\mathbf{0 . 0 0 1}$ \\
ED & $252 / 288$ & 87.5 & 10.0 & $18.668-23.332$ & \\
${ }^{*} \mathrm{O} / \mathrm{N}=$ observed death number/total patient number in each group & & $9.050-10.950$ & \\
\hline
\end{tabular}

${ }^{*} \mathrm{O} / \mathrm{N}=$ observed death number/total patient number in each group

Table 3: Multivariate analysis of risk factors for SCLC patients treated with etoposide and platinum-based chemotherapy

\begin{tabular}{lccc}
\hline Variables & Hazard ratio & $\mathbf{9 5 \%}$ CI & $\boldsymbol{P}$ value \\
\hline Gender & 0.809 & $0.670-0.976$ & $\mathbf{0 . 0 2 7}$ \\
Age & 0.985 & $0.802-1.210$ & 0.888 \\
KPS & 1.834 & $1.498-2.245$ & $<\mathbf{0 . 0 0 1}$ \\
Smoking history & 1.148 & $0.954-1.382$ & 0.145 \\
Anemia & 1.060 & $0.794-1.416$ & 0.691 \\
Lymphocyte & 0.879 & $0.729-1.059$ & 0.176 \\
NLR & 1.030 & $0.837-1.267$ & 0.780 \\
LMR & 1.053 & $0.848-1.307$ & 0.641 \\
LDH & 1.415 & $1.161-1.724$ & $\mathbf{0 . 0 0 1}$ \\
ALP & 1.002 & $0.818-1.228$ & 0.984 \\
Surgery & 0.725 & $0.429-1.226$ & 0.231 \\
Chemotherapy cycles & 0.784 & $0.660-0.931$ & $\mathbf{0 . 0 0 5}$ \\
Thoracic irradiation & 0.800 & $0.671-0.954$ & $\mathbf{0 . 0 1 3}$ \\
Metastatic sites & 1.440 & $1.073-1.934$ & $\mathbf{0 . 0 1 5}$ \\
Stage & 1.428 & $1.177-1.732$ & $<\mathbf{0 . 0 0 1}$ \\
\hline
\end{tabular}

$35.1 \%$ vs $22.9 \%, p=0.001$ ), low LMR (yes vs no, $42.1 \%$ vs $21.5 \%, p<0.001$ ), high ALP level (yes vs no, $36.2 \%$ vs $24.5 \%, p<0.001$ ), extensive disease (yes vs no, $42.0 \%$ vs $16.9 \%, p<0.001)$ and more metastatic sites at diagnosis (yes vs no, $55.4 \%$ vs $24.3 \%, p<0.001$ ). High LDH could be more frequently seen in patients with extensive disease, metastatic sites $\geq 2$, anemia, low LMR and high NLR levels.

\section{Predictive prognosis analysis for variable subgroups in patients with SCLC}

Subgroup analysis were performed to assess parameters affecting the prognosis of patients in different groups and identify which group of patients can benefit more from the treatment of chemotherapy cycles $\geq 4$ and thoracic radiotherapy. Subgroup analysis was performed in variable KPS, LDH, gender, tumor stage and metastatic sites groups based on multivariate analysis results. The parameters were the same with Table 2. Tumor stage, thoracic irradiation, metastatic sites and $\mathrm{LDH}$ were independent prognostic factors of KPS $>80$ points patients ( $p<0.05$ on uni- and multivariate analysis). Tumor stage and chemotherapy cycles were independent prognostic factors in patients with KPS $\leq 80$ points. Details of independent prognostic factors in other subgroups analysis can be seen in Table 5 .

Subgroup analysis showed that patients with male gender, KPS $\leq 80$ points, $\mathrm{LDH} \leq 216.5 \mathrm{U} / \mathrm{L}$, extensive disease and metastatic sites $<2$ can benefit more from $\geq 4$ chemotherapy cycles $(p<0.05$ on uni- and multivariate analysis, Figure 2). In addition, patients with male gender, KPS $>80$ points, $\mathrm{LDH} \leq 216.5 \mathrm{U} / \mathrm{L}$, limited disease and metastatic sites $<2$ can benefit more from 
Table 4: Correlation of the variable LDH level with the clinicopathological characteristics of SCLC patients

\begin{tabular}{|c|c|c|c|}
\hline \multirow[t]{3}{*}{ Variable } & \multicolumn{3}{|c|}{ LDH (U/L) } \\
\hline & $\leq 216.5$ & $>216.5$ & $p$ value \\
\hline & n $(\%)$ & n $(\%)$ & \\
\hline Age (years) & & & 0.051 \\
\hline$\leq 65$ & $418(74.5)$ & $143(25.5)$ & \\
\hline$>65$ & $97(66.4)$ & $49(33.6)$ & \\
\hline Gender & & & 0.449 \\
\hline Male & $335(73.8)$ & $119(26.2)$ & \\
\hline Female & $180(71.1)$ & $73(28.9)$ & \\
\hline KPS & & & 0.274 \\
\hline$>80$ points & $401(73.8)$ & $142(26.2)$ & \\
\hline$\leq 80$ points & $114(69.5)$ & $50(30.5)$ & \\
\hline Smoking history & & & 0.224 \\
\hline Yes & $315(71.3)$ & $127(28.7)$ & \\
\hline No & $200(75.5)$ & $65(24.5)$ & \\
\hline Anemia & & & 0.025 \\
\hline Yes & $39(60.9)$ & $25(39.1)$ & \\
\hline No & $476(74.0)$ & $167(26.0)$ & \\
\hline Lymphocyte $\left(\times 10^{9} / \mathrm{L}\right)$ & & & 0.426 \\
\hline$\leq 1.65$ & $267(71.6)$ & $106(28.4)$ & \\
\hline$>1.65$ & $248(74.3)$ & $86(25.7)$ & \\
\hline NLR & & & 0.001 \\
\hline$\leq 3.18$ & $356(77.1)$ & $106(22.9)$ & \\
\hline$>3.18$ & $159(64.9)$ & $86(35.1)$ & \\
\hline LMR & & & $<0.001$ \\
\hline$\leq 2.615$ & $113(57.9)$ & $82(42.1)$ & \\
\hline$>2.615$ & $402(78.5)$ & $110(21.5)$ & \\
\hline ALP (U/L) & & & 0.003 \\
\hline$\leq 119.5$ & $413(75.5)$ & $134(24.5)$ & \\
\hline$>119.5$ & $102(63.8)$ & $58(36.2)$ & \\
\hline Stage & & & $<0.001$ \\
\hline LD & $348(83.1)$ & $71(16.9)$ & \\
\hline ED & $167(58.0)$ & $121(42.0)$ & \\
\hline Metastatic sites & & & $<0.001$ \\
\hline$<2$ & $486(75.7)$ & $156(24.3)$ & \\
\hline$\geq 2$ & $29(44.6)$ & $36(55.4)$ & \\
\hline
\end{tabular}


Table 5: Suggested prognostic factors in variable subgroups of SCLC patients

\begin{tabular}{|c|c|c|c|c|c|c|c|c|c|c|}
\hline \multirow[t]{2}{*}{ Variables } & \multicolumn{2}{|c|}{ KPS (points) } & \multicolumn{2}{|c|}{ LDH (U/L) } & \multicolumn{2}{|c|}{ tumor stage } & \multicolumn{2}{|c|}{ metastatic sites } & \multicolumn{2}{|c|}{ Gender } \\
\hline & $>80$ & $\leq \mathbf{8 0}$ & $\leq 216.5$ & $>216.5$ & LD & ED & $<2$ & $\geq 2$ & male & female \\
\hline Surgery & $+/-$ & $-/ \varnothing$ & $+/-$ & $-/ \varnothing$ & $-/ \varnothing$ & $-/ \varnothing$ & $+/-$ & $\varnothing / \varnothing$ & $-/ \varnothing$ & $+/-$ \\
\hline Stage & $+/+$ & $+/+$ & $+/+$ & $+/+$ & $\varnothing / \varnothing$ & $\varnothing / \varnothing$ & $+/+$ & $\varnothing / \varnothing$ & $+/+$ & $+/+$ \\
\hline Chemotherapy cycles & $+/-$ & $+/+$ & $+/+$ & $-/ \varnothing$ & $-/ \varnothing$ & $+/+$ & $+/+$ & $-/ \varnothing$ & $+/+$ & $+/-$ \\
\hline Thoracic irradiation & $+/+$ & $+/-$ & $+/+$ & $+/-$ & $+/+$ & $+/-$ & $+/+$ & $-/ \varnothing$ & $+/+$ & $+/-$ \\
\hline Metastatic sites & $+/+$ & $-/ \varnothing$ & $+/-$ & $+/-$ & $\varnothing / \varnothing$ & $+/+$ & $\varnothing / \varnothing$ & $\varnothing / \varnothing$ & $+/+$ & $+/-$ \\
\hline Smoking history & $+/-$ & $-/ \varnothing$ & $-/ \varnothing$ & $+/+$ & $+/+$ & $-/ \varnothing$ & $+/-$ & $-/ \varnothing$ & $-/ \varnothing$ & $+/-$ \\
\hline NLR & $+/-$ & $-/ \varnothing$ & $+/-$ & $-/ \varnothing$ & $-/ \varnothing$ & $+/-$ & $+/-$ & $-/ \varnothing$ & $+/-$ & $-/ \varnothing$ \\
\hline LMR & $-/ \varnothing$ & $-/ \varnothing$ & $-/ \varnothing$ & $-/ \varnothing$ & $-/ \varnothing$ & $+/-$ & $-/ \varnothing$ & $-/ \varnothing$ & $+/-$ & $-/ \varnothing$ \\
\hline Gender & $+/-$ & $-/ \varnothing$ & $-/ \varnothing$ & $-/ \varnothing$ & $-/ \varnothing$ & $-/ \varnothing$ & $+/-$ & $-/ \varnothing$ & $-/ \varnothing$ & $-/ \varnothing$ \\
\hline Anemia & $+1-$ & $-/ \varnothing$ & $-/ \varnothing$ & $-/ \varnothing$ & $-/ \varnothing$ & $-/ \varnothing$ & $+1-$ & $-/ \varnothing$ & $-/ \varnothing$ & $-/ \varnothing$ \\
\hline $\mathrm{RBC}$ & $-/ \varnothing$ & $-/ \varnothing$ & $-/ \varnothing$ & $-/ \varnothing$ & $-/ \varnothing$ & $-/ \varnothing$ & $-/ \varnothing$ & $-/ \varnothing$ & $-/ \varnothing$ & $+/-$ \\
\hline $\mathrm{LDH}$ & $+/+$ & $+1-$ & $\varnothing / \varnothing$ & $\varnothing / \varnothing$ & $+/+$ & $+/+$ & $+/+$ & $+/+$ & $+/+$ & $+/-$ \\
\hline ALP & $+/-$ & $-/ \varnothing$ & $-/ \varnothing$ & $-/ \varnothing$ & $-/ \varnothing$ & $-/ \varnothing$ & $+/-$ & $-/ \varnothing$ & $-/ \varnothing$ & $-/ \varnothing$ \\
\hline Age & $-/ \varnothing$ & $-/ \varnothing$ & $+/-$ & $-/ \varnothing$ & $+/-$ & $-/ \varnothing$ & $+/-$ & $-/ \varnothing$ & $-/ \varnothing$ & $-/ \varnothing$ \\
\hline PS & $\varnothing / \varnothing$ & $\varnothing / \varnothing$ & $+/+$ & $+/+$ & $+/+$ & $+/+$ & $+/+$ & $-/ \varnothing$ & $+/+$ & $+/+$ \\
\hline WBC & $-/ \varnothing$ & $-/ \varnothing$ & $-/ \varnothing$ & $+/+$ & $-/ \varnothing$ & $-/ \varnothing$ & $-/ \varnothing$ & $+/+$ & $-/ \varnothing$ & $-/ \varnothing$ \\
\hline Family history & $-/ \varnothing$ & $-/ \varnothing$ & $-/ \varnothing$ & $-/ \varnothing$ & $+/+$ & $-/ \varnothing$ & $-/ \varnothing$ & $-/ \varnothing$ & $-/ \varnothing$ & $-/ \varnothing$ \\
\hline Lymphocytes & $-/ \varnothing$ & $-/ \varnothing$ & $-/ \varnothing$ & $+/-$ & $-/ \varnothing$ & $-/ \varnothing$ & $-/ \varnothing$ & $-/ \varnothing$ & $-/ \varnothing$ & $-/ \varnothing$ \\
\hline Neutrophils & $-/ \varnothing$ & $-/ \varnothing$ & $-/ \varnothing$ & $+/+$ & $-/ \varnothing$ & $-/ \varnothing$ & $-/ \varnothing$ & $-/ \varnothing$ & $-/ \varnothing$ & $-/ \varnothing$ \\
\hline
\end{tabular}

$+/+=$ significant on both univariate and multivariate analysis; $+/-=$ significant on univariate analysis, not significant on multivariate analysis; $-/ \varnothing=$ not significant on univariate analysis and not assessed on multivariate analysis; $\varnothing / \varnothing=$ not studied, the parameters used were the same with Table 2 and factors with no statistical significance in variable subgroups were not shown.

thoracic irradiation (Figure 3). Patients with PS $>80$ points and female gender can get survival benefits from more cycles of chemotherapy on univariate analysis $(p<0.05)$, but it holds no significance on multivariate analysis. Patients with $\mathrm{LDH}>216.5 \mathrm{U} / \mathrm{L}$, limited disease and metastatic sites $\geq 2$ can get little benefits from more cycles of chemotherapy ( $p>0.05)$. Patients with KPS $\leq 80$ points, $\mathrm{LDH}>216.5 \mathrm{U} / \mathrm{L}$, female gender and extensive disease can benefit from thoracic irradiation on univariate analysis $(p<0.05)$, but it holds no significant difference on multivariate analysis. Patients with metastatic sites $\geq 2$ can get little benefit from the treatment of chemotherapy cycles $\geq 4$ and thoracic irradiation $(p>0.05)$.

\section{DISCUSSION}

In our study, we find that female patients, KPS $>80$ points, $\mathrm{LDH} \leq 216.5 \mathrm{U} / \mathrm{L}$, chemotherapy cycles $\geq 4$, thoracic irradiation, metastatic sites $<2$ and limited disease are independent positive prognostic factors for SCLC treated with etoposide and platinum-based chemotherapy. Selected patients can benefit more from the treatment of chemotherapy cycles $\geq 4$ and thoracic radiotherapy. Patients with male gender, KPS $\leq 80$ points, $\mathrm{LDH} \leq 216.5 \mathrm{U} / \mathrm{L}$, extensive disease and metastatic sites $<2$ can benefit more from $\geq 4$ cycles of chemotherapy. And patients with male gender, KPS $>80$ points, $\mathrm{LDH} \leq 216.5 \mathrm{U} / \mathrm{L}$, limited disease and metastatic sites $<2$ can benefit more from thoracic radiotherapy. Inflammatory markers of NLR and LMR are associated with the prognosis of SCLC patients, but it holds no statistical significance on multivariate and subgroup analysis. Serum LDH, which can be easily available, still has an independent prognostic values in the condition of more parameters and these results are valuable in clinics.

SCLC is a fatal disease characterized as early recurrence and rapid progression. Many patients progressed quickly and died soon even they received 
the same treatment strategies. So the identification of patients who can benefit more from the same treatment strategy is important. $\mathrm{LDH}<216.5 \mathrm{U} / \mathrm{L}$ and metastatic sites $<2$ reflect a low tumor burdens. Subgroup analysis shows chemotherapy cycles $\geq 4$ has more significant role in patients with tolerated performance status, male gender, low tumor burdens and relatively extensive disease. Thoracic radiotherapy is more effective in the treatment of patients with better tolerance, limited features, male gender and low tumor burdens. Patients with $\mathrm{LDH}<216.5 \mathrm{U} / \mathrm{L}$, male gender and metastatic sites $<2$ can significantly benefit from $\geq$ cycles of chemotherapy and thoracic irradiation. The overall survival benefits of patients with metastatic sites $\geq 2$ got from these two treatment is limited, and the treatment in these cases should be carefully considered. Female gender can benefit from these two treatment strategies, but no statistical significance is found on multivariate analysis. Maybe the sample size and grouping of bias can partly account for this result, and the roles of these treatment in female gender still need to be verified. These results can help us identify which treatment strategy is more suitable for variable groups of patients. In addition, it can avoid some additional toxic reactions for patients who can benefit little from $\geq 4$ cycles of chemotherapy and thoracic irradiation. This is important for a long-term survival and better quality of life.

Inflammation plays an important role in cancer development, and inflammatory markers of NLR, PLR, LMR et al have been demonstrated to be associated with the prognosis of various malignancies $[10,11,15-21]$. White blood cell (WBC) counts can reflect body inflammatory status, and it plays limited role in the prognosis of the whole SCLC patients $(p>0.05)$. However, subgroup analysis shows WBC counts $>10 \times 10^{9} / \mathrm{L}$ is a positive independent prognostic factor for patients with $\mathrm{LDH}>216.5 \mathrm{U} / \mathrm{L}$ and metastatic sites $\geq 2$. WBC may have an important role in patients with high tumor burdens and advanced stage. Similar phenomenon can also be found in previous study and the mechanism remains unknown [1]. High NLR and low LMR are associated with the prognosis of SCLC patients with the presence of more parameters, but no statistical significance is found on multivariate analysis. High NLR reflects a status of neutrophilia or lymphocytopenia. Vascular endothelial growth factor (VEGF), as a proangiogenic factor, is mainly secreted by neutrophils and it plays a critical role in cancer progression [22]. Lymphocytopenia indicates a poor body lymphocyte mediated immune status to cancer. Recent study reveals high NLR is correlated with a distinct cytokine profile related to key biological processes involved in carcinogenesis including an increased expression of interleukin 6 (IL-6), IL-8 and VEGF et al [23]. High NLR and low LMR can promote tumor angiogenesis and inhibit body immunity, leading to a poor prognosis. The potential roles of these inflammatory markers still need to be verified in further research.

$\mathrm{LDH}$ has been identified as prognostic factor of SCLC and various malignancies in previous studies [1,5-8,16,21,24-29]. However, the mechanism remains poorly understood. In our study, we found, taking into account of more parameters, LDH still holds statistical significance on a series of analysis. High LDH level
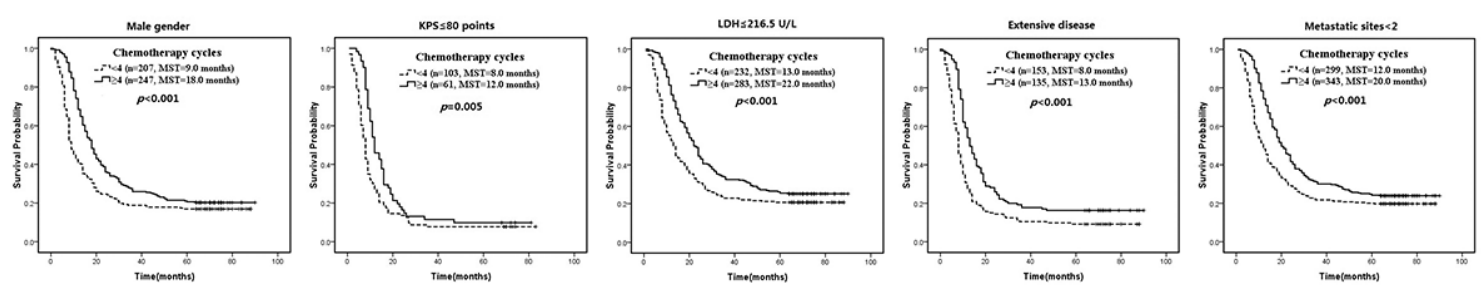

Figure 2: Patients with male gender, KPS $\leq \mathbf{8 0}$ points, $\mathbf{L D H} \leq \mathbf{2 1 6}$. 5U/L, extensive disease and metastatic sites $<2$ can benefit more from the management of etoposide and platinum-based chemotherapy ( $<<0.05$ on uni- and multivariate analysis). MST, median survival time.
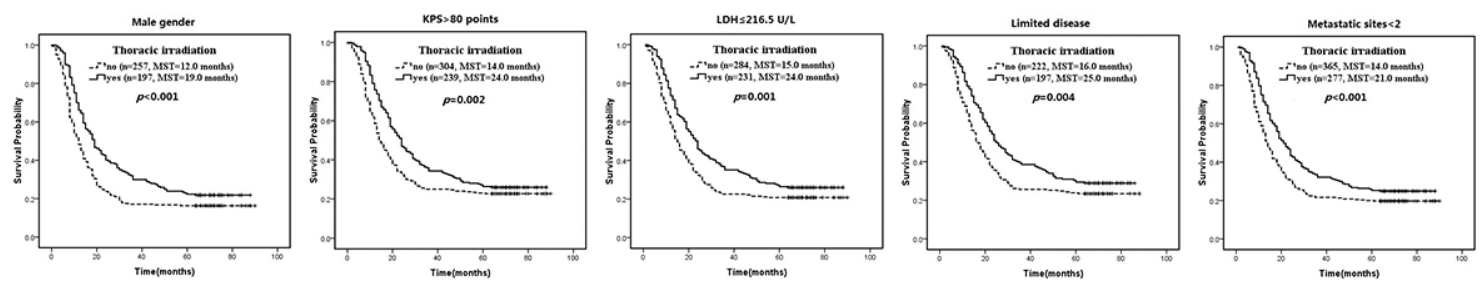

Figure 3: Patients with male gender, KPS $>\mathbf{8 0}$ points, $\mathbf{L D H} \leq \mathbf{2 1 6}$. 5U/L, limited disease and metastatic sites $<2$ can benefit more from the treatment of thoracic irradiation ( $\mathrm{p}<0.05$ on uni- and multivariate analysis). MST, median survival time. 
is a negative independent prognostic factors of SCLC patients. LDH plays an important role in energy production in various cell types, and an elevated LDH level may promote tumor progression by regulating tumor metabolism [29]. High LDH also reflects a higher tumor burden, the extent of disease and rapid turnover of tumor cells [5,29]. In our study, high LDH is also found to be associated with high ALP level, and patients with elevated ALP show worse treatment tolerance and response in the analysis of overall survival and progression free survival (PFS) [26,29]. In addition, elevated ALP is also correlated with a high tumor burden and can promote tumor progression [29]. Patients with $\mathrm{LDH} \leq 216.5 \mathrm{U} / \mathrm{L}$ can significantly benefit from the treatment of $\geq 4$ cycles of chemotherapy and thoracic irradiation on subgroup analysis. These results may have some hints for our clinical decisions for the treatment of patients with variable LDH levels.

There are some limitations of our study. First, it is a single constitutional retrospective analysis and we exclude many patients treated with best supportive care or other treatment strategies in order to assess the prognostic roles of parameters in patients treated with etoposide and platinum-based chemotherapy. That is why there are more limited disease patients in our cohort. Second, we do not assess the impact of these factors on PFS for the absence of PFS information in some patients. In addition, patients number in some groups is relatively small. We will pay more attention to these details in our further study.

In conclusion, female patients, KPS $>80$ points, $\mathrm{LDH} \leq 216.5 \mathrm{U} / \mathrm{L}, \quad$ chemotherapy $\quad$ cycles $\geq 4$, thoracic irradiation, metastatic sites $<2$ and limited disease are independent positive prognostic factors for patients treated with etoposide and platinum-based chemotherapy. Patients with male gender, KPS $\leq 80$ points, $\mathrm{LDH} \leq 216.5 \mathrm{U} / \mathrm{L}$, extensive disease and metastatic sites $<2$ can benefit more from $\geq 4$ cycles of chemotherapy. And patients with male gender, KPS $>80$ points, $\mathrm{LDH} \leq 216.5 \mathrm{U} / \mathrm{L}$, limited disease and metastatic sites $<2$ can benefit more from thoracic radiotherapy. Further retrospective and prospective studies are urgently needed to provide evidence-based recommendations for our clinics.

\section{MATERIALS AND METHODS}

\section{Patients}

We conducted a retrospective analysis on 1126 cases with pathologically confirmed SCLC who were registered and followed up in our hospital between January 2008 and January 2010. Only patients treated with etoposide and platinum-based chemotherapy were included in this study. The criteria for entry into our study were as follows: 1. histologically or cytologically proved SCLC; 2 . no prior anti-tumor therapies; 3 . age $\leq 75$ years; 4 . complete follow-up information; 5 . combined routine blood test and liver function examinations. Finally, 707 patients were included in our study. Our study was approved by the Ethics Committee of Harbin Medical University Cancer Hospital and written informed consent was obtained from each patient. Limited disease (LD) is defined as disease confined to the ipsilateral chest within a single radiation field, while extensive disease (ED) was defined as disease beyond the ipsilateral hemithorax including malignant pleural, pericardial effusion, or hematogenous metastasis.

\section{Data collection}

The following parameters were collected and evaluated for prognostic impact: age ( $\leq 65$ or $>65$ years), gender, KPS (Karnofsky Performance Status), smoking history, family history of cancer, serum red blood cell (RBC), platelet $(\leq 300,000$ or $>300,000 / \mu 1)$, white blood cell (WBC, $\leq 10$ or $\left.>10 \times 10^{9} / \mathrm{L}\right)$, hemoglobin, neutrophil $\left(\leq 4.95\right.$ or $\left.>4.95 \times 10^{9} / \mathrm{L}\right)$, lymphocyte $\left(\leq 1.65\right.$ or $\left.>1.65 \times 10^{9} / \mathrm{L}\right)$, monocyte $(\leq 0.495$ or $\left.>0.495 \times 10^{9} / \mathrm{L}\right)$, NLR $(\leq 3.18$ or $>3.18)$, PLR $(\leq 176.5$ or $>176.5)$, LMR $(\leq 2.615$ or $>2.615)$, aspartate aminotransferase (AST, $\leq 40$ or $>40 \mathrm{U} / \mathrm{L}$ ), alanine aminotransferase (ALT, $\leq 40$ or $>40 \mathrm{U} / \mathrm{L}), \mathrm{LDH}(\leq 216.5$ or $>216.5 \mathrm{U} / \mathrm{L}), \operatorname{ALP}(\leq 119.5$ or $>119.5 \mathrm{U} / \mathrm{L})$, albumin $(\leq 40$ or $>40 \mathrm{~g} / \mathrm{L})$, globulin $(\leq 35$ or $>35 \mathrm{~g} / \mathrm{L}$ ), surgery, chemotherapy cycles, thoracic irradiation, metastatic sites and tumor stage at initial diagnosis. A low serum RBC level was defined as a red blood cell count $<3.5$ or $4.0 \times 10^{9} / \mathrm{L}$ in females and males, respectively. Anemia is defined as a hemoglobin value $<12.0 \mathrm{~g} / \mathrm{dl}$ in men and $11.0 \mathrm{~g} / \mathrm{dl}$ in women. The NLR, PLR, and LMR values were calculated using the neutrophil, platelet, lymphocyte and monocyte counts as the ratio of neutrophil and platelet counts to lymphocyte or lymphocyte counts to monocyte, respectively.

\section{Statistical analysis}

Mean values \pm standard deviation (SD) or median and range were calculated for continuous variables. The optimal cut-off point for neutrophil, lymphocyte, monocyte, NLR, PLR, LMR, LDH and ALP were calculated by a receiver operating characteristic (ROC) curve analysis. Survival outcomes were dichotomized by survival status (alive or dead) until a defined time of the median survival time of 15.0 months in the ROC curve analysis. The overall survival time was defined from diagnosis to death or the last follow-up of April 2015 if the patients were still alive. The survival curves and 95\% confidence intervals (CIs) were obtained using the Kaplan-Meier method, and the survival was compared by log-rank test. The relationship between LDH and the clinicopathological features were evaluated using the chi-squared test. Univariate analysis was used to examine the potential prognostic roles of all factors. The prognostic factors with $p$-value $\leq 0.05$ in the univariate analysis were examined in the multivariate analysis. SPSS 16.0 statistical software was used for statistical analysis. All $P$-values $\leq 0.05$ were considered statistically significant. 


\section{ACKNOWLEDGMENTS}

This work was supported by National Natural Scientific Foundation of China (No: 81572824, 81673007 and 81672931), Health Department of Heilongjiang Provincial of China (No: 2011-124), Harbin Medical University Cancer Hosptial major project Foundation (No: JJZ-2010-01), Provincial Department of Education (No: 12521284).

\section{CONFLICTS OF INTEREST}

None declared.

\section{REFERENCES}

1. Kawahara M, Fukuoka M, Saijo N, Nishiwaki Y, Ikegami H, Tamura T, Shimoyama M, Suemasu K, Furuse K. Prognostic factors and prognostic staging system for small cell lung cancer. Jpn J Clin Oncol. 1997;27:158-65.

2. Lally BE, Urbanic JJ, Blackstock AW, Miller AA, Perry MC. Small cell lung cancer: have we made any progress over the last 25 years? Oncologist. 2007;12:1096-104.

3. Osterlind K, Andersen PK. Prognostic factors in small cell lung cancer: multivariate model based on 778 patients treated with chemotherapy with or without irradiation. Cancer Res. 1986;46:4189-94.

4. Maestu I, Pastor M, Gómez-Codina J, Aparicio J, Oltra A, Herranz C, Montalar J, Munárriz B, Reynés G. Pretreatment prognostic factors for survival in small-cell lung cancer: a new prognostic index and validation of three known prognostic indices on 341 patients. Ann Oncol. 1997;8:547-53.

5. Cerny T, Blair V, Anderson H, Bramwell V, Thatcher N. Pretreatment prognostic factors and scoring system in 407 small-cell lung cancer patients. Int J Cancer. 1987;39:146-9.

6. Souhami RL, Bradbury I, Geddes DM, Spiro SG, Harper PG, Tobias JS. Prognostic significance of laboratory parameters measured at diagnosis in small cell carcinoma of the lung. Cancer Res. 1985;45:2878-82.

7. Rawson NS, Peto J. An overview of prognostic factors in small cell lung cancer. A report from the subcommittee for the management of lung cancer of the United Kingdom coordinating committee on cancer research. Br J Cancer. 1990;61:597-604.

8. Dearing MP, Steinberg SM, Phelps R, Anderson MJ, Mulshine JL, Ihde DC, Johnson BE. Outcome of patients with small-cell lung cancer: effect of changes in staging procedures and imaging technology on prognostic factors over 14 years. J Clin Oncol. 1990;8:1042-9.

9. Paesmans M, Sculier JP, Lecomte J, Thiriaux J, Libert P, Sergysels R, Bureau G, Dabouis G, Van Cutsem O, Mommen P, Ninane V, Klastersky J. Prognostic factors for patients with small cell lung carcinoma: analysis of a series of 763 patients included in 4 consecutive prospective trials with a minimum follow-up of 5 years. Cancer. 2000;89:523-33.

10. Mantovani A, Allavena P, Sica A, Balkwill F. Cancer-related inflammation. Nature. 2008;454:436-44.

11. Shalapour S, Karin M. Immunity, inflammation, and cancer: an eternal fight between good and evil. J Clin Invest. 2015;125:3347-55.

12. Fridman WH, Pagès F, Sautès-Fridman $\mathrm{C}$, Galon J. The immune contexture in human tumours: impact on clinical outcome. Nat Rev Cancer. 2012;12:298-306.

13. Loi S, Sirtaine N, Piette F, Salgado R, Viale G, Van Eenoo F, Rouas G, Francis P, Crown JP, Hitre E, de Azambuja E, Quinaux E, Di Leo A, et al. Prognostic and predictive value of tumorinfiltrating lymphocytes in a phase III randomized adjuvant breast cancer trial in node-positive breast cancer comparing the addition of docetaxel to doxorubicin with doxorubicin-based chemotherapy: BIG 02-98. J Clin Oncol. 2013;31:860-7.

14. Halama N, Michel S, Kloor M, Zoernig I, Benner A, Spille A, Pommerencke T, von Knebel DM, Folprecht G, Luber B, Feyen N, Martens UM, Beckhove P, et al. Localization and density of immune cells in the invasive margin of human colorectal cancer liver metastases are prognostic for response to chemotherapy. Cancer Res. 2011;71:5670-7.

15. Wu G, Yao Y, Bai C, Zeng J, Shi D, Gu X, Shi X, Song Y. Combination of platelet to lymphocyte ratio and neutrophil to lymphocyte ratio is a useful prognostic factor in advanced non-small cell lung cancer patients. Thorac Cancer. 2015;6:275-87.

16. Go SI, Kim RB, Song HN, Kang MH, Lee US, Choi HJ, Lee SJ, Cho YJ, Jeong YY, Kim HC, Lee JD, Kim SH, Kang JH, et al. Prognostic significance of the lymphocyteto-monocyte ratio in patients with small cell lung cancer. Med Oncol. 2014;31:323.

17. Yamagishi T, Fujimoto N, Nishi H, Miyamoto Y, Hara N, Asano M, Fuchimoto Y, Wada S, Kitamura K, Ozaki S, Kishimoto T. Prognostic significance of the lymphocyteto-monocyte ratio in patients with malignant pleural mesothelioma. Lung Cancer. 2015;90:111-7.

18. Zhang GM, Zhu Y, Luo L, Wan FN, Zhu YP, Sun LJ, Ye DW. Preoperative lymphocyte-monocyte and plateletlymphocyte ratios as predictors of overall survival in patients with bladder cancer undergoing radical cystectomy. Tumour Biol. 2015;36:8537-43.

19. Ying HQ, Deng QW, He BS, Pan YQ, Wang F, Sun HL, Chen J, Liu X, Wang SK. The prognostic value of preoperative NLR, d-NLR, PLR and LMR for predicting clinical outcome in surgical colorectal cancer patients. Med Oncol. 2014;31:305.

20. Jia W, Wu J, Jia H, Yang Y, Zhang X, Chen K, Su F. The Peripheral Blood Neutrophil-To Lymphocyte Ratio Is Superior to the Lymphocyte-To-Monocyte Ratio for Predicting the Long-Term Survival of Triple Negative Breast Cancer Patients. PLoS One. 2015;10:e143061. 
21. Wang $\mathrm{X}$, Jiang $\mathrm{R}$, Li K. Prognostic significance of pretreatment laboratory parameters in combined small-cell lung cancer. Cell Biochem Biophys. 2014;69:633-40.

22. Kusumanto YH, Dam WA, Hospers GA, Meijer C, Mulder NH. Platelets and granulocytes, in particular the neutrophils, form important compartments for circulating vascular endothelial growth factor. Angiogenesis. 2003;6: 283-7.

23. Z-Y Chen, K Raghav, C H Lieu, Z-Q Jiang, C Eng, J-N Vauthey, G J Chang, W Qiao, J Morris, D Hong, P Hoff, H Tran, D G Menter, et al. Cytokine profile and prognostic significance of high neutrophil-lymphocyte ratio in colorectal cancer. $\mathrm{Br} \mathrm{J}$ Cancer. 2015; 112: 1088-97.

24. Hermes A, Gatzemeier U, Waschki B, Reck M. Lactate dehydrogenase as prognostic factor in limited and extensive disease stage small cell lung cancer-A retrospective single institution analysis. Respir Med. 2010;104:1937-42.

25. Weide B, Elsässer M, Büttner P, Pflugfelder A, Leiter U, Eigentler TK, Bauer J, Witte M, Meier F, Garbe C. Serum markers lactate dehydrogenase and $\mathrm{S} 100 \mathrm{~B}$ predict independently disease outcome in melanoma patients with distant metastasis. Br J Cancer. 2012;107:422-8.

26. Maisano R, Azzarello D, Del Medico P, Maisano M, Bottari M, Egitto G, Nardi M. Alkaline phosphatase levels as a prognostic factor in metastatic colorectal cancer treated with the FOLFOX 4 regimen: a monoinstitutional retrospective study. Tumori. 2011;97:39-42.

27. Sonpavde G, Pond GR, Berry WR, de Wit R, Armstrong AJ, Eisenberger MA, Tannock IF. Serum alkaline phosphatase changes predict survival independent of PSA changes in men with castration-resistant prostate cancer and bone metastasis receiving chemotherapy. Urol Oncol. 2012;30:607-13.

28. Neofytou K, Giakoustidis A, Mudan S. CALy Prognostic Score (CEA, ALP, Lymphocyte Count) as a predictor of survival after hepatectomy for liver-only colorectal metastases. J BUON. 2015;20:746-55.

29. Wei XL, Zhang DS, He MM, Jin Y, Wang DS, Zhou YX, Bai L, Li ZZ, Luo HY, Wang FH, Xu RH. The predictive value of alkaline phosphatase and lactate dehydrogenase for overall survival in patients with esophageal squamous cell carcinoma. Tumour Biol. 2016;37:1879-87. 